\title{
Near Field in the Vicinity of Wireless Base-Station Antennas: An Exposure Compliance Approach
}

\author{
Sebastián Blanch, Jordi Romeu, Member, IEEE, and Angel Cardama, Member, IEEE
}

\begin{abstract}
Great social concern has risen about the potential health hazard of living near a cellular telephony base-station antenna, and certain technical questions have been posed on the appropriate way to measure exposure in its vicinity. In this paper, standard spherical near-near field transformation is proposed to obtain the electromagnetic field close to the antenna in free space conditions. The field obtained in this way allows to define an exclusion zone from the exposure compliance point of view, but also makes possible to bound the error committed by standard field measurement procedures. Furthermore, the visualization of the electromagnetic field in the proximity of the antenna in free space conditions, allows to define clearance templates that have to be met in the siting of the antenna in complex environments in order not to obstruct its main beam.
\end{abstract}

Index Terms-Antenna measurements, land mobile radio equipment, public safety.

\section{INTRODUCTION}

$\mathbf{O}$ VER the past few years the widespread use of cellular telephony has resulted in the ubiquitous presence of cellular base stations. In certain countries of Europe, a social concern has risen about possible health hazards resulting from living near a base station. In dense urban areas the deployment of wireless networks is sometimes even jeopardized by social opposition to the siting of base stations. As a consequence of this social concern, several groups of experts have been asked to examine possible effects of mobile phones and base stations on health [1], [2]. On the other hand, in July 1999, the European Commission issued a recommendation relative to electromagnetic exposure [3]. This recommendation follows the INCIRP guidelines [4] and are equivalent to the IEEE standards [5]. In the United States, the Federal Communication Commission (FCC) has provided a guide to assist local government officials in devising efficient procedures for assuring that antenna facilities comply with FCC limits for human exposure to radiofrequency [6]. Human exposure is quantified in terms of the specific absorption rate (SAR) within the exposed body.

The computation of the SAR from the incident electromagnetic field requires complex computations when the incident field differs from a plane wave. In [7], a hybrid approach has

Manuscript received June 22, 2001; revised November 27, 2001. This work was funded in part by CICYT and the European Commission under Grant TIC2001-2364-C03-01, and in part by the Departament d'Universitats, Recerca i Societat de la Informacio (DURSI), Generalitat de Catalunya.

The authors are with the Department of Signal Theory and Communications, Telecommunication Engineering School of the Universitat Politecnica de Catalunya and Centre Tecnologic de Telecomunicacions de Catalunya (CTTC), Barcelona, Spain.

Publisher Item Identifier S 0018-926X(02)05447-9. been used to compute the SAR due to exposure to a base-station antenna electromagnetic radiation. In [7] antenna radiation is approximated by its far-field radiation pattern. In a complex environment the exposure is strongly nonuniform. Nevertheless, it is shown that the maximum E-field, set by the guidelines, offers appropriate protection to human exposure in terms of the SAR. There is not a reported case in which the E-field is within the guidelines, but the SAR limit is exceeded. A recent paper [8] has suggested an efficient method to compute the SAR in complex environments close to the antenna siting. However, the problem still remains in the accurate on-site measurement of the incident field. As discussed in [9], commercial field probes operating in the wireless communication bands are sensitive to $|\vec{E}|^{2}$, and the reading is usually expressed in $V / m$. These instruments are referred as isotropic E-field probes. In the far-field region of the antenna, where only tangential components of the fields are present, and $\mathrm{H}$-field is linked to the E-field through the free space impedance, the isotropic E-field probe is a useful instrument in determining not only incident field, but also the magnitude of the Poynting vector. In this case, $\wp=|\vec{E}|^{2} / \eta$, where $\wp$ is the magnitude of the Poynting vector and $\eta$ is the free space impedance. In the vicinity of the antenna there is not such a direct relationship between electric and magnetic field. On the basis of the difficulty in measuring near fields and their dependence on the environment, certain authors recommend that people should not live in the near field of an antenna [10]. The far field limit is defined by the usual $2 D^{2} / \lambda$ criteria.

Nevertheless, the near field of the base-station antenna can be accurately determined. In [9], it is analytically shown for a particular type of antenna, i.e., in phase fed collinear arrays, how the intercepted power by a cylinder enclosing the antenna varies according to the radius of the cylinder. Present base-station antennas are more complex structures. For sector antennas, that is, with a beamwidth on the horizontal plane between $60^{\circ}$ and $85^{\circ}$, they are usually formed by eight or ten radiating elements, stacked vertically and typically spaced between 0.6 and $0.75 \lambda$. The elements are usually microstrip patches, or dipoles over a ground plane. The feeding of the elements is appropriately weighted:

- to produce an electrical down tilt of the main beam between $2^{\circ}$ and $8^{\circ}$;

- to reduce the sidelobes in the broadside direction to diminish interference to adjacent cells;

- to obtain pattern null-filling in the coverage region.

Due to the complex weighting of the elements of these more complex antennas, the near-field may exhibit strong variations 


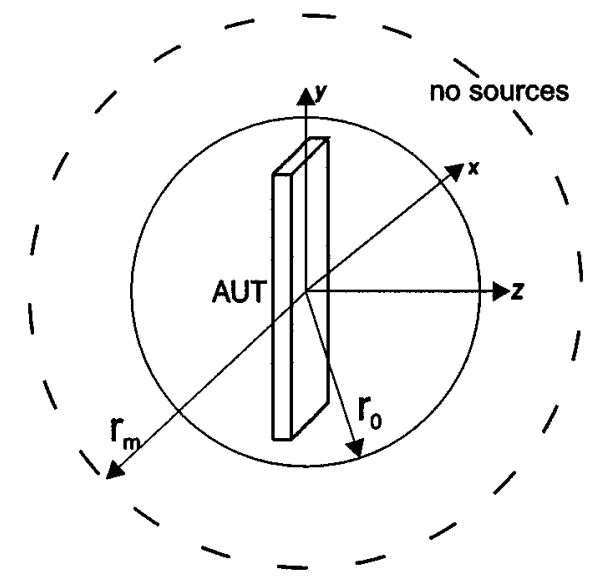

Fig. 1. Spherical near-field measurement scheme.

in its vicinity. In this paper it is proposed to employ spherical mode decomposition of the near-field radiated by a base-station antenna to obtain the electromagnetic fields in its vicinity. In Section II, the theoretical considerations concerning spherical near-near transformation are reviewed. In Section III, results for two typical base-station antennas are presented and it is shown the degree of error committed in estimating the magnitude of the Poynting vector assuming plane-wave conditions. It is also shown that an exclusion zone around the base station can be easily defined. For common cellular applications and considering the exposure levels set in the guidelines, this exclusion zone is much smaller than the far field criteria proposed by certain authors. Finally, the representation of the magnitude of the Poynting vector in the vicinity of the base-station antenna in free space conditions provides a valuable tool for the correct definition of clearance templates in order not to impair the performance of the antenna when sited in a complex environment. In Section IV, the relevant conclusions of the paper are presented.

\section{THEORETICAL CONSIDERATIONS}

Spherical near to far field transformation is a standard antenna measurement technique employed to obtain the antenna far-field pattern from a near-field measurement [11]. The near-field measurement can also be employed to obtain the electromagnetic field in the vicinity of the antenna. Let us consider the measurement scheme of Fig. 1, where $r_{0}$ is the radius of the minimum sphere that encloses the antenna and $r_{m}$ the measurement distance. The fields in a source-free region outside a radiating antenna can be written as a weighted sum of the spherical wave functions

$$
\begin{aligned}
\vec{E}(r, \theta, \phi) & =\frac{k}{\sqrt{\eta}} \sum_{s=1}^{2} \sum_{n=1}^{\infty} \sum_{m=-n}^{n} Q_{s m n} \vec{F}_{s, m, n}(r, \theta, \phi) \\
\vec{H}(r, \theta, \phi) & =-i k \sqrt{\eta} \sum_{s=1}^{2} \sum_{n=1}^{\infty} \sum_{m=-n}^{n} Q_{s m n} \vec{F}_{3-s, m, n}(r, \theta, \phi)
\end{aligned}
$$

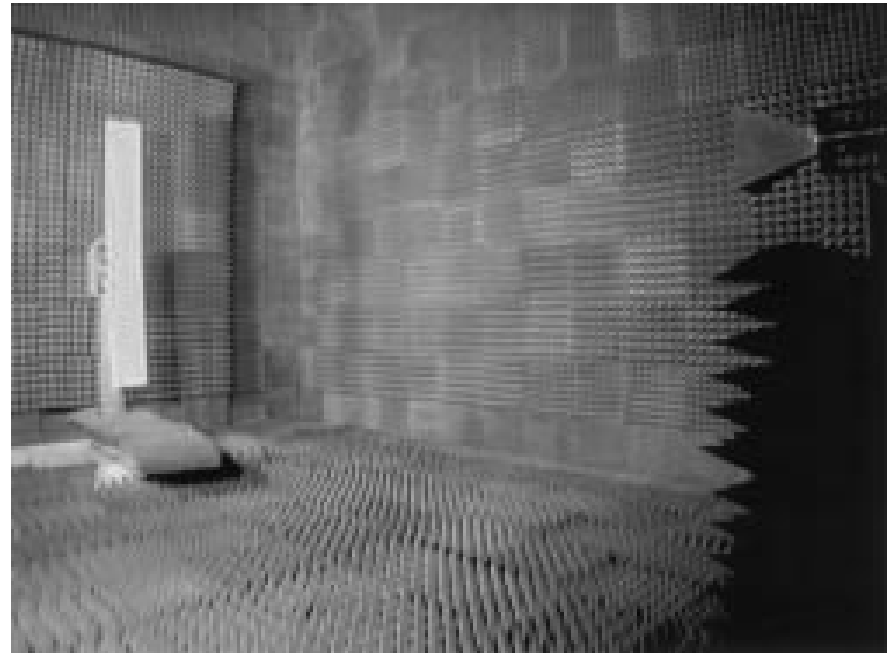

Fig. 2. GSM base-station antenna placed in the anechoic chamber for measurement. Distance between antenna and probe is $5 \mathrm{~m}$.

where the functions $\vec{F}_{s m n}$ are defined as

$$
\begin{aligned}
\vec{F}_{1 m n}(r, \theta, \phi)= & \frac{1}{\sqrt{2 \pi}} \frac{1}{\sqrt{n(n+1)}}\left(-\frac{m}{|m|}\right)^{m} \\
& \times\left\{h_{n}^{2}(k r) \frac{i m \bar{P}_{n}^{|m|}(\cos \theta)}{\sin \theta} e^{i m \phi} \hat{\theta}\right. \\
& \left.-h_{n}^{2}(k r) \frac{d \bar{P}_{n}^{|m|}(\cos \theta)}{d \theta} e^{i m \phi} \hat{\phi}\right\} \\
\vec{F}_{2 m n}(r, \theta, \phi)= & \frac{1}{\sqrt{2 \pi}} \frac{1}{\sqrt{n(n+1)}}\left(-\frac{m}{|m|}\right)^{m} \\
& \times\left\{\frac{n(n+1)}{k r} h_{n}^{2}(k r) \bar{P}_{n}^{|m|}(\cos \theta) e^{i m \phi} \hat{r}\right. \\
& +\frac{1}{k r} \frac{d}{d(k r)}\left\{k r h_{n}^{2}(k r)\right\} \\
& \times \frac{d \bar{P}_{n}^{|m|}(\cos \theta)}{d \theta} e^{i m \phi} \hat{\theta} \\
& +\frac{1}{k r} \frac{d}{d(k r)}\left\{k r h_{n}^{2}(k r)\right\} \\
& \left.\times \frac{i m \bar{P}_{n}^{|m|}(\cos \theta)}{\sin \theta} e^{i m \phi} \hat{\phi}\right\}
\end{aligned}
$$

and $\bar{P}_{n}^{|m|}$ are the associated Legendre functions and $h_{n}^{2}(k r)$ the spherical Hankel function of the second kind. In a real case, the number of modes for the convergence of the series is finite, and the index $n$ is truncated to $N$, where $N$ is given by the empirical rule

$$
N \approx k r_{0}+10
$$

where $r_{0}$ is the radius of the minimum sphere that encloses the antenna. All the coefficients $Q_{s m n}$ can be determined from the knowledge of either $\vec{E}$ or $\vec{H}$ on the measurement sphere. This is true even for $r_{m} \rightarrow \infty$ (far field) where the radial componentes of the fields vanish. The details of theory and practice of spherical near to far field transformation can be found in [11]. 

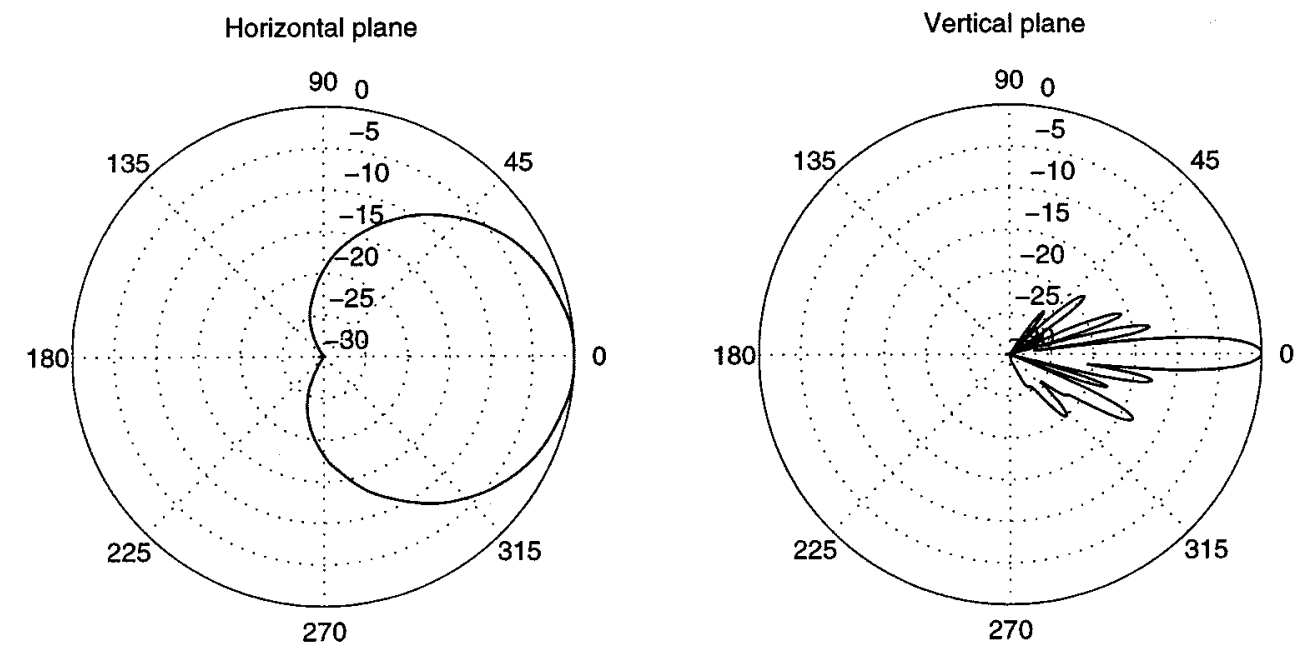

Fig. 3. Main cuts of the radiation pattern of the GSM antenna.

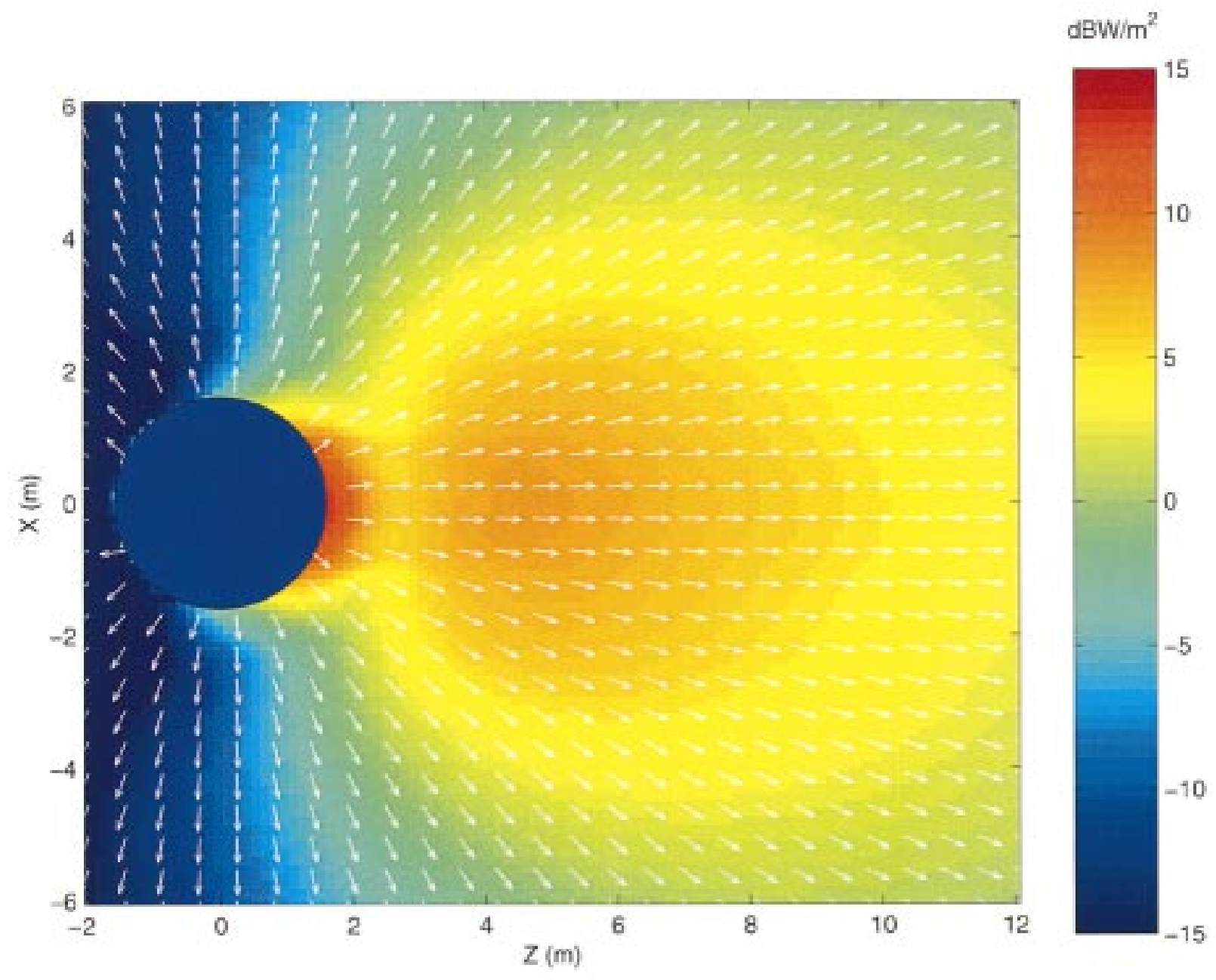

Fig. 4. Poynting vector in the vicinity of a GSM base-station antenna. Data has been computed from a spherical near-field measurement. The radiated power is $60 \mathrm{~W}$.

\section{Results AND APPLICATION}

In order to show the validity of this approach, two example cellular base-station antennas have been measured. The experimental setup is shown in Fig. 2. It is essentially an anechoic chamber for antenna measurements where the distance between the antenna under test and the measurement probe is $5 \mathrm{~m}$. The measurement procedure is the same that is followed in standard spherical near-field measurements for antenna pattern determination. The near field is sampled over a sphere that encloses the 


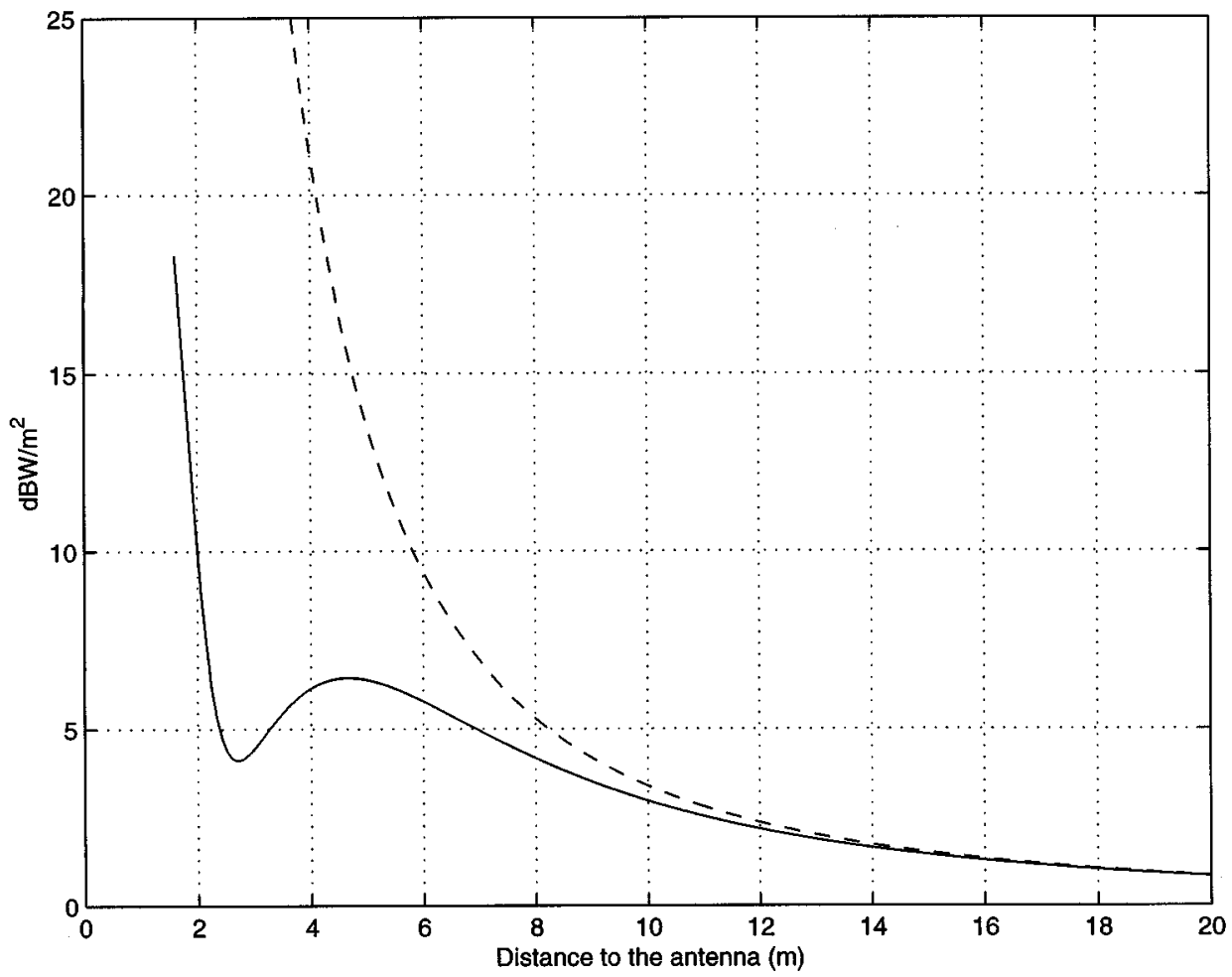

Fig. 5. Solid line is the magnitude of the Poynting vector for a GSM antenna as a function of the distance along the main beam direction. The dashed line shows the far-field approximation given by $E I R P / 4 \pi r^{2}$. The radiated power is $60 \mathrm{~W}$.

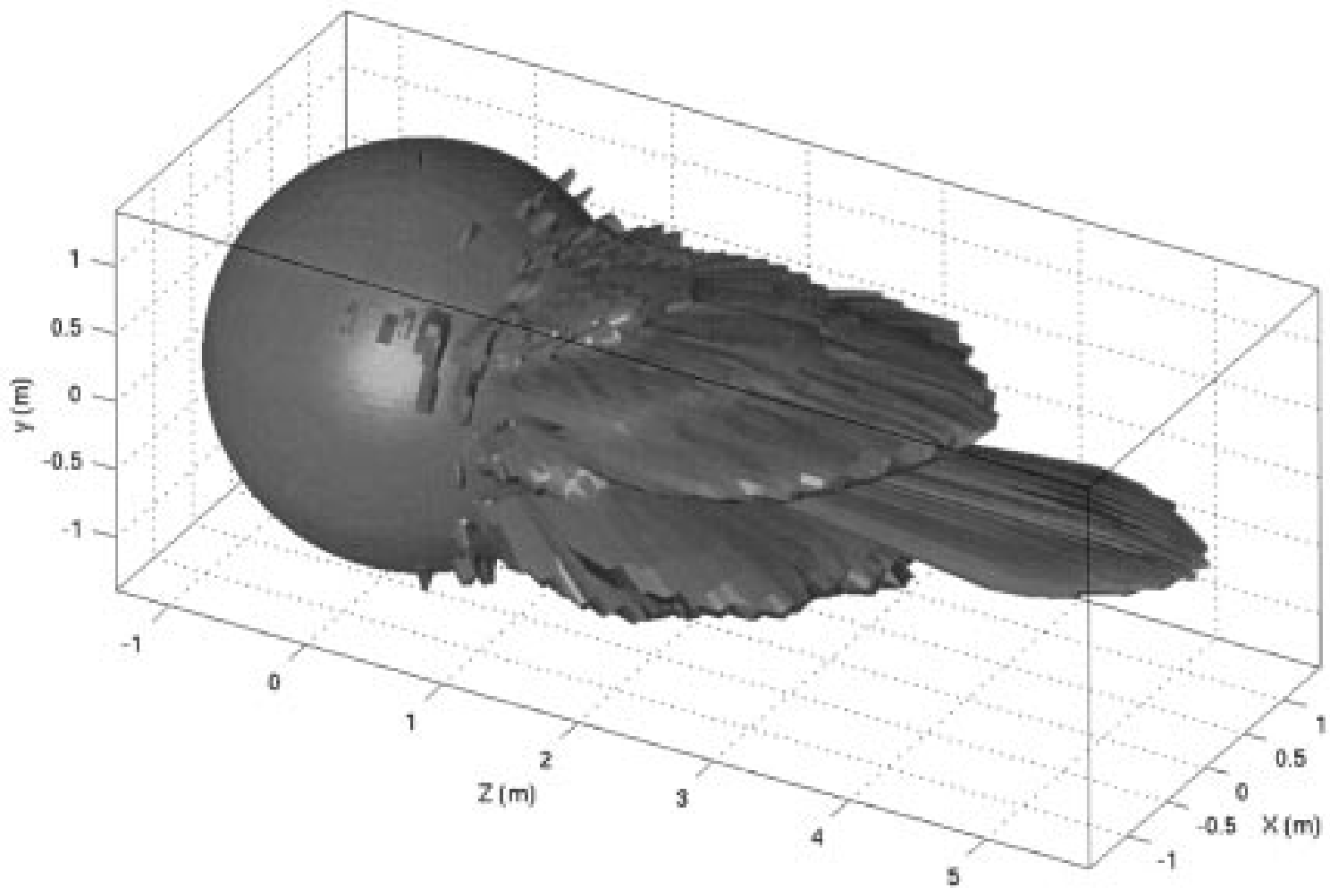

Fig. 6. Volume where the power density exceeds $6 \mathrm{~W} / \mathrm{m}^{2}$ for $60 \mathrm{~W}$ of radiated power for the GSM antenna.

AUT (antenna under test). Once the coefficients $Q_{s m n}$ are determined from the near-field measurement, the radiated field in any point outside the sphere that encloses the AUT is found following (1). 


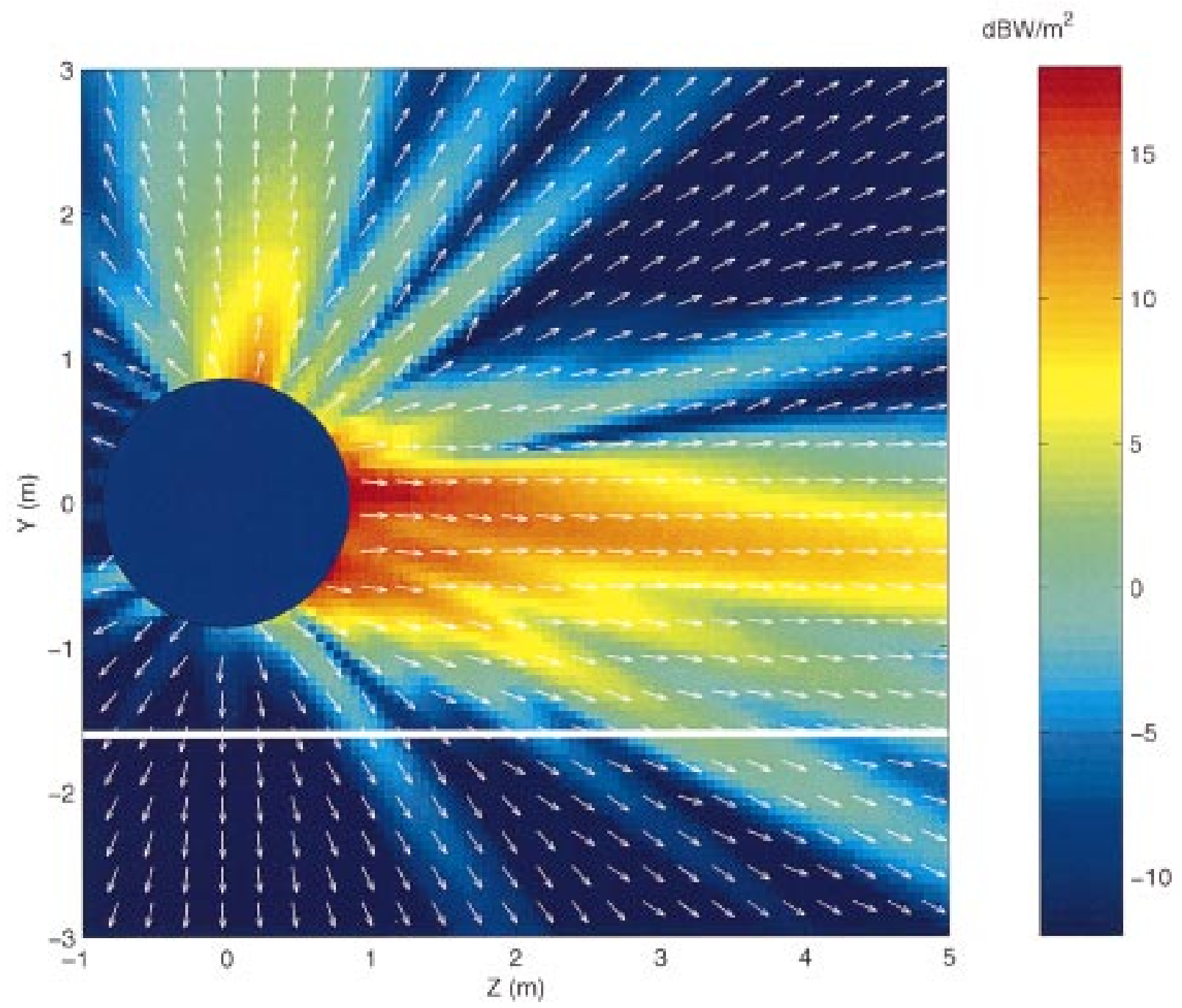

Fig. 7. Poynting vector in the vicinity of the DCS base-station antenna in the vertical plane. The radiated power is $60 \mathrm{~W}$.

Two sector base antennas have been tested to demonstrate the potential use of this technique. One antenna operates in the $900-\mathrm{MHz}$ band, and the second in the $1800-\mathrm{MHz}$ band. Both antennas exhibit similar characteristics. The directivity is of the order of $17 \mathrm{dBi}$, and the $-3-\mathrm{dB}$ beamwidth in the horizontal plane is $65^{\circ}$. The dimensions of both antennas are similar in terms of the wavelength. The antennas are approximately $7 \lambda$ in height and $1.2 \lambda$ in width. The $900-\mathrm{MHz}$ antenna is designed for the European GSM wireless system, and $1800-\mathrm{MHz}$ for the European DCS wireless system. The DCS antenna has an electrical downtilt of $6^{\circ}$, while the GSM antenna has the maximum in the broadside direction. Both antennas are representative of sector base-station antennas employed in cellular wireless systems. These antennas are eight-element linear arrays of microstrip patch antennas.

First of all, the results for the GSM antenna are presented. In order to have a better understanding of the antenna characteristics the main cuts of far-field pattern are presented in Fig. 3. This far-field pattern has been obtained through spherical near to far-field transformation. The procedure described in this paper has been applied to compute the near field in the vicinity of the antenna. Once all the components of the electric and magnetic field are known, the Poynting vector in each point is easily computed as $\Re\left\{\vec{E} \times \vec{H}^{*}\right\}$. In Fig. 4, the Poynting vector is shown for a radiated power of $60 \mathrm{~W}$. The color scale depicts its magnitude and the arrows its direction. The plot shows the Poynting vector in the horizontal plane, that is the $x z$ plane according to the geometry of Fig. 1. The blue circle corresponds to the minimum sphere that encloses the antenna where the fields cannot be computed. Fig. 4 clearly shows how the power density is distributed in the vicinity of the antenna. As the distance increases the Poynting vector has a dominant radial component, and hence the power density has a $1 / r^{2}$ dependence.

In Fig. 5 the magnitude of the Poynting vector in the direction of maximum radiation is plotted as a function of the distance. For comparison the far-field estimation of the radiated power density given by $E I R P / 4 \pi r^{2}$ is also plotted. Notice that for this antenna the far-field distance given by the usual $2 D^{2} / \lambda$ criteria is of the order of $30 \mathrm{~m}$, but for distances greater than $10 \mathrm{~m}$ an upper bound for the radiated power density can be computed using the antenna far-field parameters.

Another application of the proposed methodology is the determination of an exclusion volume. The exclusion volume is defined by the volume where the power density exceeds the 


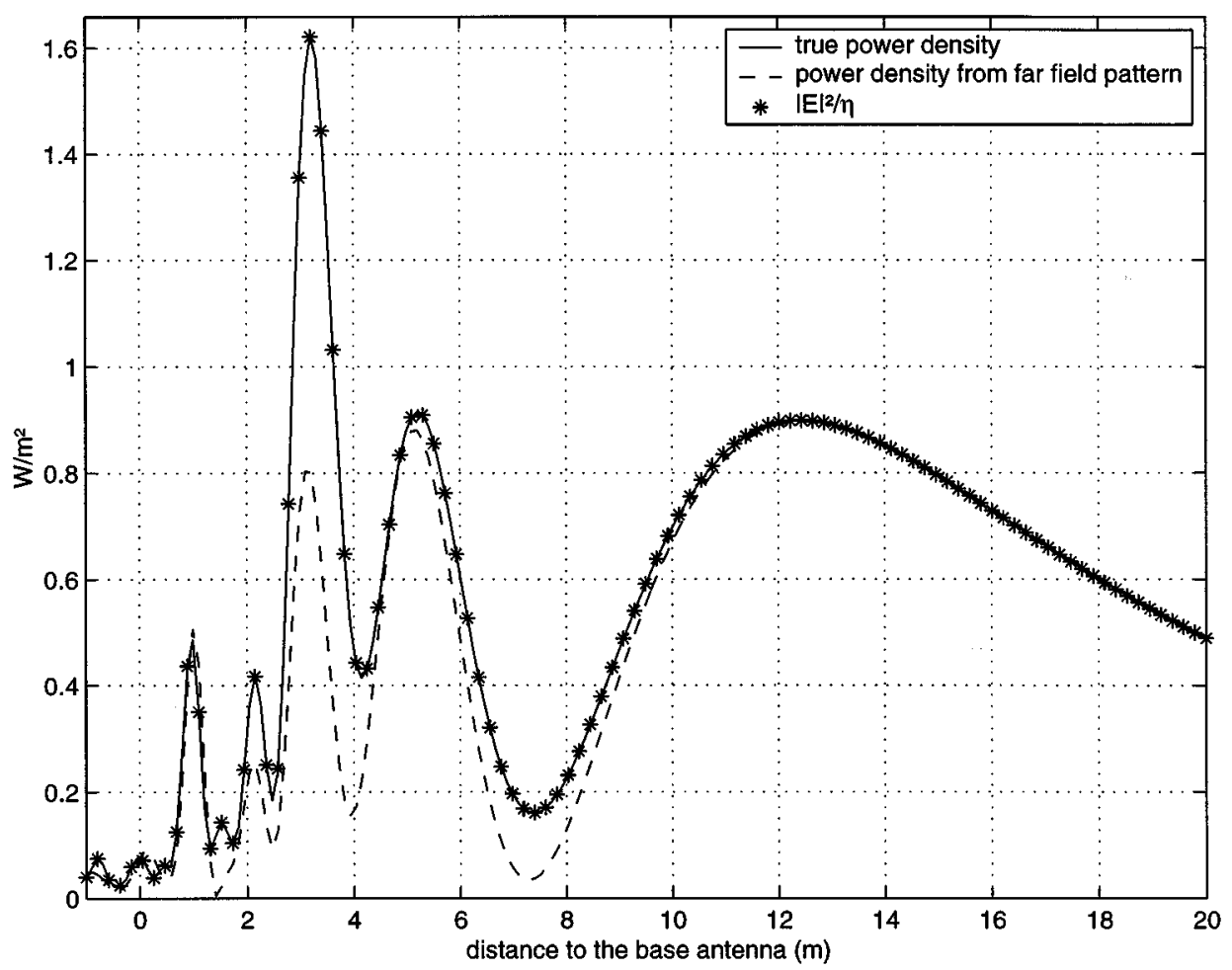

Fig. 8. Plot of the magnitude of the Poynting vector as a function of the distance to the base-station antenna. The data is plotted at a distance of $1.6 \mathrm{~m}$ below the center of the antenna (white line in Fig. 7. The solid line is the true value obtained according the procedure described in this paper. The stars show $|\vec{E}|^{2} / \eta$, that is the value that would be obtained from an isotropic E-field probe. The dashed line shows the value obtained as the far-field approximation given by $E I R P(\theta, \phi) / 4 \pi r^{2}$.

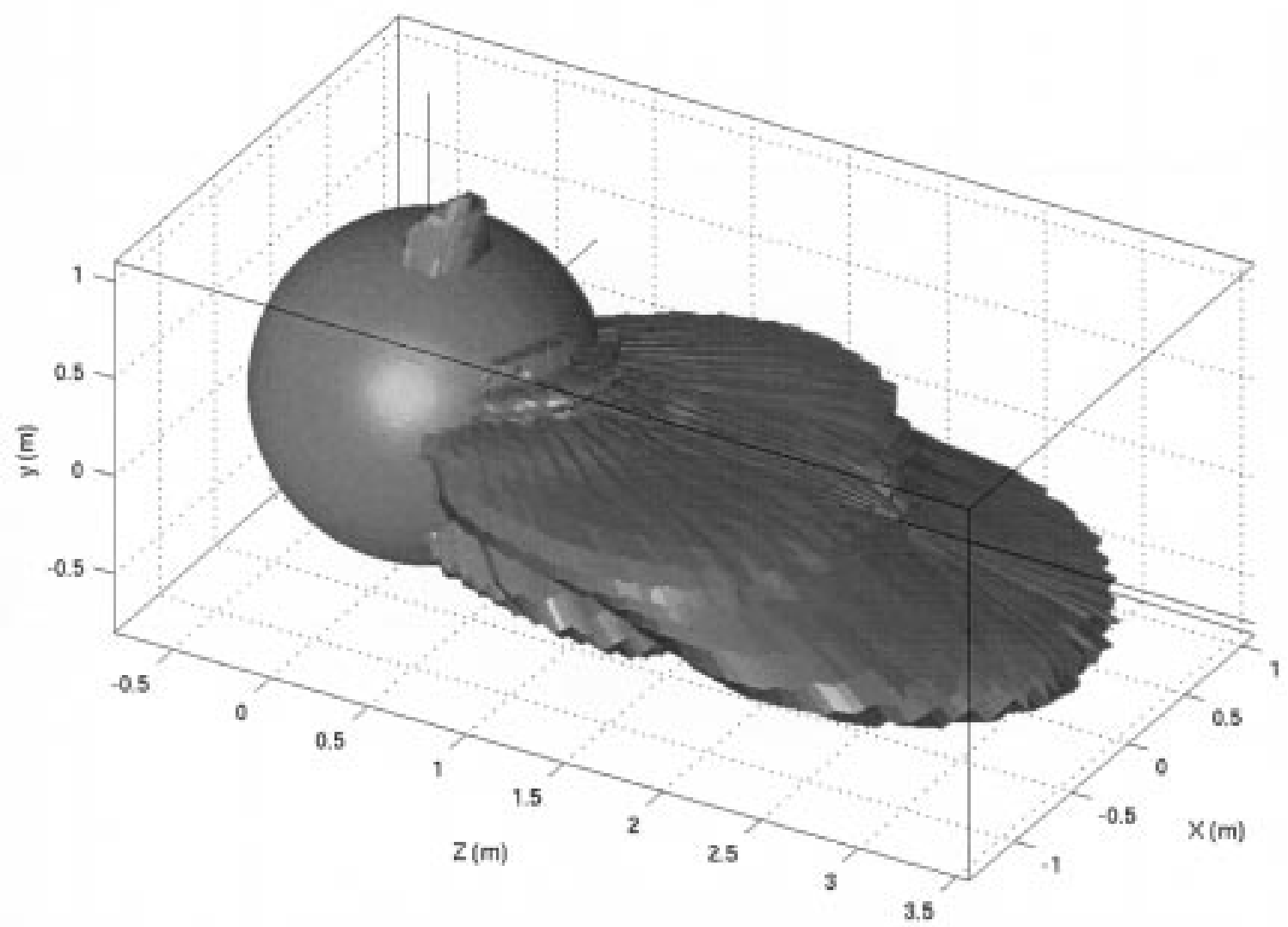

Fig. 9. Volume where the power density exceeds $12 \mathrm{~W} / \mathrm{m}^{2}$ for $60 \mathrm{~W}$ of radiated power. 
value given by the exposure guidelines. Therefore, it is assumed that the siting of the antenna must guarantee that the public cannot intrude this exclusion volume. In Fig. 6, a three-dimensional (3-D) representation of the exclusion volume is depicted for this antenna and a radiated power of $60 \mathrm{~W}$. In this case, the exclusion volume is defined for a maximum value of the power density of $6 \mathrm{~W} / \mathrm{m}^{2}$. This is the ANSI-IEEE exposure guideline for uncontrolled environments at $900 \mathrm{MHz}$ [5]. For this particular case and antenna the exclusion volume can be enclosed by a box of $6 \mathrm{~m} \times 3 \mathrm{~m} \times 3 \mathrm{~m}$.

A second example antenna is a $1800-\mathrm{MHz}$ DCS antenna. This antenna has an elecrical downtilt of $6^{\circ}$. Fig. 7 shows the Poynting vector in the vertical plane, that is the $y z$ plane according to the geometry of Fig. 1. In this case also a radiated power of 60-W has been considered. The effect of the electrical downtilt can be appreciated clearly. It is also clear that when the antenna is placed $2 \mathrm{~m}$ above the roof of a building its effect on the near-field distribution is very low, since the incident field on the roof is about $20 \mathrm{~dB}$ lower than in the main-beam direction.

In Fig. 8, a plot of the magnitude of the Poynting vector as a function of the distance of the base-station antenna is presented. The distance is taken along the $z$ axis and $1.6 \mathrm{~m}$ below the center of the antenna (white line in Fig. 7). It has been computed in three different ways. In solid line, the magnitude of the Poynting vector computed for a $60 \mathrm{~W}$ radiated power and following the procedure proposed in this paper. This is denoted as true power density. For comparison, the stars show the value of $|\vec{E}|^{2} / \eta$ where $|\vec{E}|$ is the magnitude of the electric field as it would be measured by an isotropic E-field probe and $\eta$ is the free space impedance. A closer look of Fig. 8 would reveal that errors smaller than $1 \%$ are made in obtaining the magnitude of the Poyting vector by this latter procedure. Finally, the dashed line shows the magnitude of the Poynting vector if it is estimated from the antenna far-field pattern. In this latter case, the error is much larger and the magnitude of the Poynting vector is underestimated in certain cases. Good agreement is only obtained when the distance approaches the far-field criteria, that is, about $15 \mathrm{~m}$. Therefore, it is not a good practice to use far-field data in estimating radiofrequency exposure in the vicinity of a base-station antenna.

Finally, in Fig. 9 it is shown the exclusion volume for this antenna for a radiated power of $60 \mathrm{~W}$. In this case, the guidelines establish an exposure limit of $12 \mathrm{~W} / \mathrm{m}^{2}$ for uncontrolled environments [5]. For this antenna and radiated power the exclusion volume can be fitted into a box of $4 \mathrm{~m} \times 3 \mathrm{~m} \times 2 \mathrm{~m}$.

\section{CONCLUSION}

In this paper, it has been shown that the near field in the vicinity of a base-station antenna can be computed from standard spherical near-field measurement. Since this is a common technique employed by antenna designers and manufacturers to obtain the far-field pattern, the application of this methodology can be easily incorporated in the final testing of base-station antennas. The results also show that on-site testing with an E-field isotropic probe provides a very good estimate of the power density in the vicinity of the antenna. On the contrary, the direct application of the far-field pattern to compute the near-field power density may result in an underestimation of the real magnitude of the power density. Finally, the methodology can be employed to define exclusion zones from the point of view of exposure compliance, but also in order not to impair the proper operation of the antenna by obstructing its near field.

\section{ACKNOWLEDGMENT}

The authors would like to thank Telefónica Móviles who provided the antennas.

\section{REFERENCES}

[1] S. W. Chairman. (2000) Mobile Phones and Health. Independent Expert Group on Mobile Phones. [Online]. Available: http://www.iegmp.org.uk/IEGMPtxt.htm

[2] (2001) Les Téléphones Mobiles, Leurs Stations de Base et la Santé, Rapport au Directeur Generale de la Santé. [Online]. Available: http://www.sante.gouv.fr

[3] "Council recommendation of 12 July 1999 on the limitation of exposure of the general public to electromagnetic fields ( $0 \mathrm{~Hz}$ to $300 \mathrm{Ghz})$," Official J. European Community L1999, vol. 59.

[4] "INCIRP Guidelines for limiting exposures to time-varying electric magnetic fields (up to $300 \mathrm{GHz}$ )," Health Phys., vol. 74, no. 4, pp. 494-522, Apr. 1998.

[5] Standard for Safety Levels With Respect to Human Exposure to RF Electromagnetic Fields $3 \mathrm{kHz}$ to $300 \mathrm{GHz}$, IEEE Std. C95.1-1999.

[6] A Local Government Official's Guide to Transmitting Antenna RF Emission Safety: Rules, Procedures, and Practical Guidance, Federal Communication Commission/Local and State Government Advisory Committee, 2000.

[7] P. Bernardi, M. Caragnaro, S. Pisa, and E. Piuzzi, "Human exposure to radio base-station antennas in urban environment," IEEE Trans. Microwave Theory Tech., vol. 48, pp. 1996-2002, Nov. 2000.

[8] G. Lazzi and O. P. Gandhi, "A mixed FDTD-integral equation approach for on-site safety assessment in complex electromagnetic environments," IEEE Trans. Antennas Propagat., vol. 48, pp. 1830-1836, Dec. 2000

[9] A. Faraone, R. Yew-Siow Tay, K. H. Joyner, and Q. Balzano, "Estimation of the average power density in the vicinity of cellular base-sation collinear array antennas," IEEE Trans. Veh. Technol., vol. 49, pp. 984-996, May 2000.

[10] A. Vander Vorst, M. D. Taurisano, and B. Stockbroeckx, "Cellular telephones: Hazards or not?," in Proc. IEEE MTT-S Symp., 2000, pp. 937-940.

[11] J. E. Hansen, Ed., Spherical Near-Field Antenna Measurements. Stevenage, U.K.: Peregrinus, 1988.

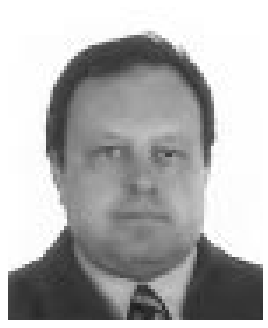

Sebastián Blanch was born in Barcelona, Spain, in 1961. He received the Ingeniero and Doctor Ingeniero degrees in telecommunication engineering, both from the Polytechnic University of Catalonia (UPC), Barcelona, Spain, in 1989 and 1996, respectively.

In 1989, he joined the Electromagnetic and Photonics Engineering Group in the Department of Signal Theory and Communications, UPC, where he is currently an Associate Professor. His research interests include antenna near-field measurements, antenna diagnostics, and antenna design. 


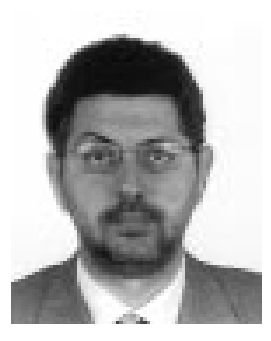

Jordi Romeu (S'88-M'93) was born in Barcelona, Spain, in 1962. He received the Ingeniero and Doctor Ingeniero degrees in telecommunication engineering, both from the Polytechnic University of Catalonia (UPC), Barcelona, Spain, in 1986 and 1991, respectively.

In 1985, he joined the Electromagetic and Photonics Engineering Group in the Department of Signal Theory and Communications at UPC, where he is currently an Associate Professor engaged in research in antenna near-field measurements, antenna diagnostics, and antenna design. In 1999, he was a Vsiting Scholar in the Antenna Lab, University of California, Los Angeles, under a NATO Scientific Program scholarship. He has published papers in the fields of antenna near-field measurements and diagnostics, and antenna design, and holds several patents.

In 1998, Dr. Romeu was Grand Winner of the European IT prize awarded by the European Commission for his contributions in the development of fractal antennas.

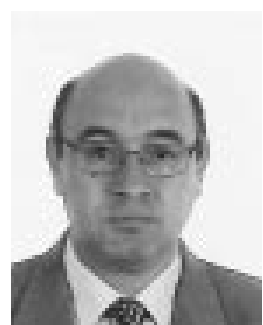

Angel Cardama (S'67-M'73) was born in Santiago de Compostela, Spain, in 1944. He received the Ingeniero de Telecomunicación degree from the Universidad Politécnica de Madrid, and the Sc.M. and $\mathrm{Ph} . \mathrm{D}$. degrees in electrical engineering from Brown University, Providence, RI, in 1968, 1970, and 1973, respectively.

In 1972, he joined the faculty of the Telecommunication Engineering School at the Polytechnic University of Catalonia, Barcelona, Spain, where he holds the position of Professor. His research interests range from the development of analytical and numerical techniques in electromagnetics to the design of microwave imaging systems and radar and communications antennas. 